Lynn, V. L., M. D. Sohigian, and E. A. Crocker (Feb. 15, 1964), Radar observations of the moon at a wavelength of 8.6 millimeters, J. Geo. Phys. Res. 69, No. 4, 781-783.

Tveten, L. H. (Mar--April, 1961), Ionospheric motions observed with high frequency backscatter sounders, J. Res. NBS 65D (Radio Prop.), No. 2, 115-127.

Tveten, L. H., L. L. Melanson, and H. G. Moller (March 1965), High Frequency Backscatter Studies, RADC Technical Report RADCTR $-65-39$.

\section{Discussion Following Davis et al.'s Paper}

I. Shapiro: In view of the extreme interest in accurate cross section measurements at long wavelengths, would it be possible to calibrate on a test sphere in orbit?

Answer: Yes, it would be useful to do so. We have looked at the Echo II satellite, but signals were too weak to be very helpful.
A. Giraud: Do you have trouble with meteor trails? Answer: Only in certain special situations and then we exclude the data.

T. Hagfors: Can you explain the variations in range of up to 2 msec?

Answer: We are currently exploring ray-tracing techniques.

G. H. Pettengill: Do you have a criterion for accepting or rejecting data which eliminates the possibility of personal bias?

Answer: Not with certainty.

T. Hagfors: How is the antenna gain measured?

Answer: Using measurements of scale models.

\title{
Lunar Mapping by Coherent-Pulse Analysis
}

\author{
T. W. Thompson \\ Cornell University
}

\author{
Arecibo Ionospheric Observatory, ${ }^{1}$ Arecibo, Puerto Rico
}

This paper describes a study of lunar radar echoes by a delay-frequency analysis which resulted in radar scattering maps of the moon. Figure 1 shows the moon as it was illuminated by the narrow antenna beam of the Arecibo Ionospheric Observatory radar. The echoes are sampled so the Doppler-shifted frequency spectrum of each delay was determined. Because of the finite length of the transmitter pulse length, the return at a particular delay must come from the range annulus $D$. Because the frequency processing admits a finite bandwidth, the return at a particular frequency must come from the frequency annulus $F$. Thus a return at a given range and frequency must come from the intersection of these two annuli, the points $P$ and $P^{\prime}$ of figure 1 . Since the

${ }^{1}$ Operated by Cornell University with the support of the Advanced Research Projects Agency under a research contract with the Air Force Office of Scientific Research, OAR.

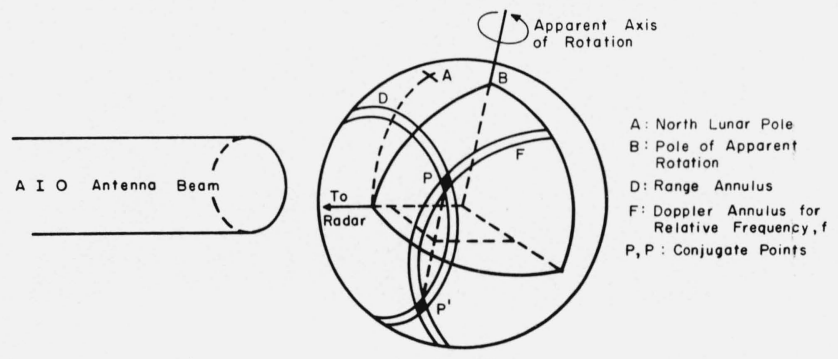

FIGURE 1. Geometry of the moon-radar system. area $P$ was within the antenna beam, the return must come from it and not from $P^{\prime}$.

In the radar scattering maps, the returns in range and frequency were mapped onto standard moon maps. These returns were expected to vary in range and frequency because of certain geometric effects, gain variation across the antenna beam, and the expected decay of reflected power with delays. These predicted variations were eliminated from the maps by using, instead of measured reflected power, the quantity

$$
\text { constant } x\left(\frac{P}{P_{0}}\right)
$$

where $P$ is the measured power, $P_{0}$ is the expected power based on the position of the reflecting area in the antenna beam and the known reflected power behavior of the moon. For convenience, the constant was chosen to make the numbers on the maps be near 100. The radar scattering maps then show normalized power returns plotted over their respective reflecting areas.

The result of one such radar mappings is shown in figure 2. The background mesh shows the delays and frequencies at which the returns were recorded. The numbers represent the normalized power returns. In the lower right-hand corner, the reflecting area for a single return is seen to be a parallelogram centered on an intersection of the mesh. The distances noted just above this parallelogram are typical for the resolution of our measurements. 


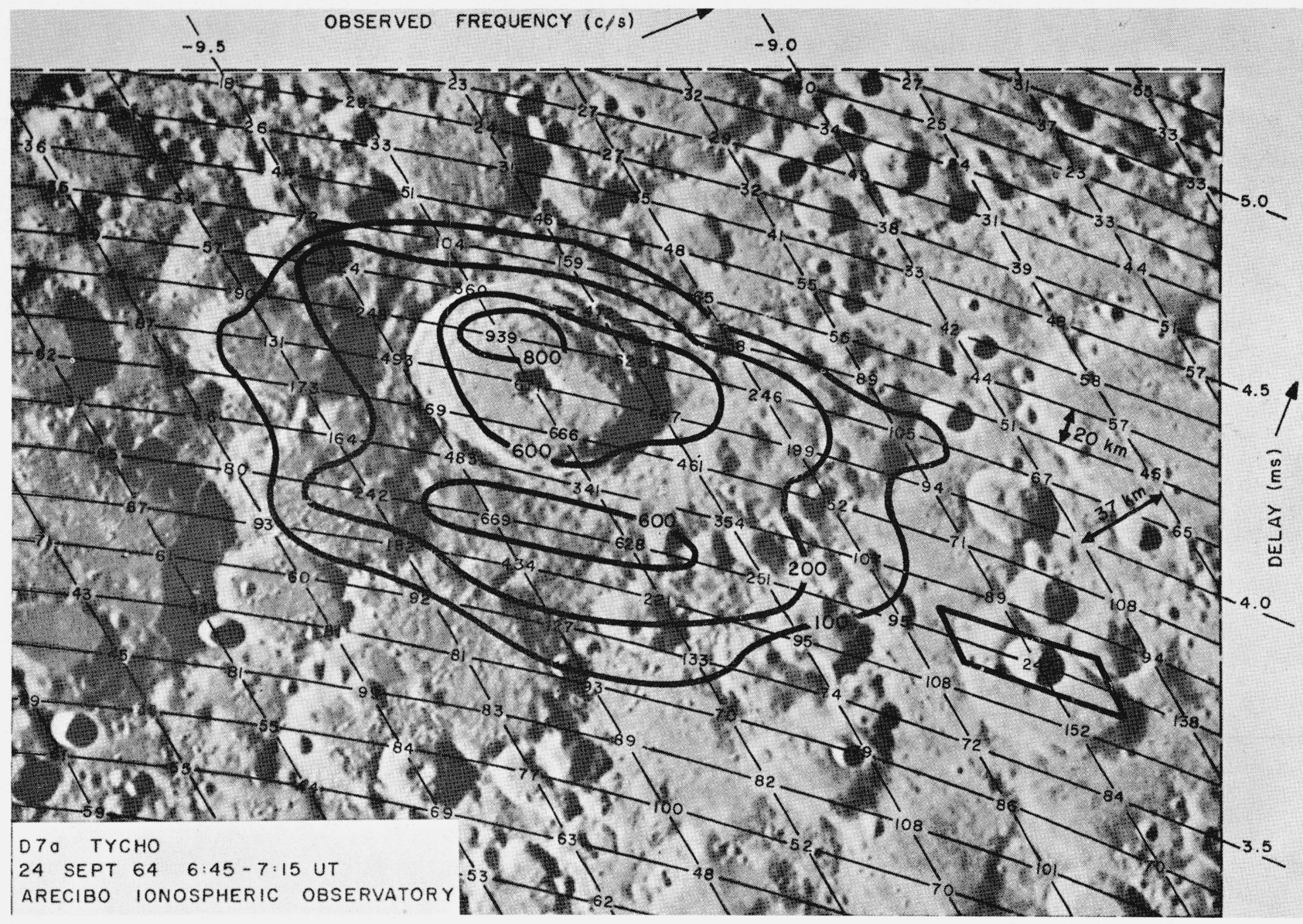

Figure 2. Contours of scattering enhancements of crater Tycho.

Two scattering enhancements are shown in figure 2 . The first is due to the small crater Heinsius A inside the parallelogram in the lower right-hand corner. The second is from the crater Tycho located in the center of the picture. Because Tycho showed enhancements at a number of delays and frequencies, contours of constant power were drawn. The power reflected by the back rim of Tycho is 18 times as great as typical areas in its environs. The interior of the crater reflected 12 times as much power as typical areas outside the center. Although the highest returns come from inside the crater, there is a high reflecting "halo" around the crater.

The study carried out last year at AIO showed that the craters which exhibited enhancements were young and rayed craters, the youngest craters on the moon. Based on theoretical estimates, the observed results suggest the enhancements are the result of localized areas of rough, exposed, and compacted rock.

Also, the craters which show radar enhancements always appear bright under full-moon illumination. This is shown in figure 3 , which shows a full-moon picture of the area shown in the radar map of figure 2 .

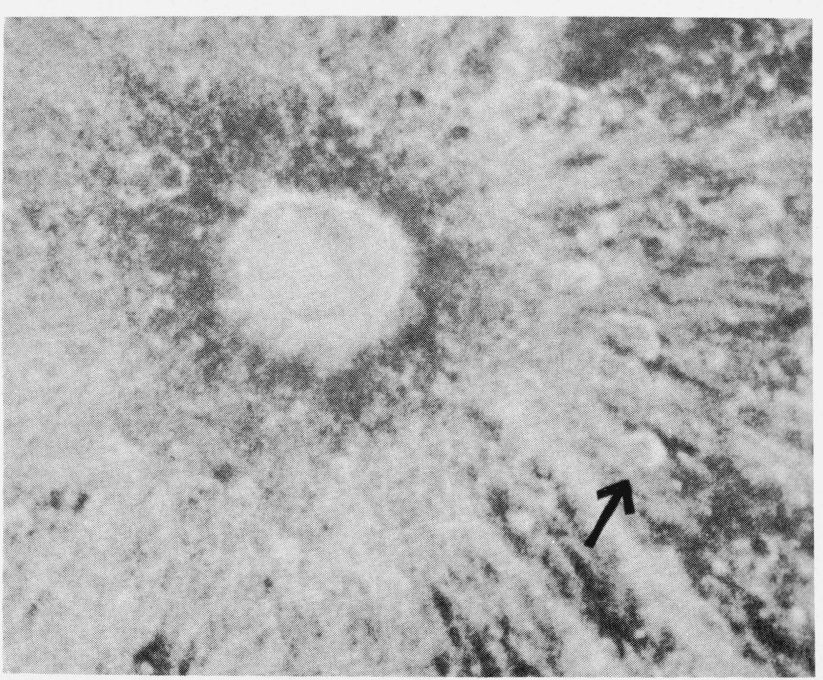

FigURE 3. Full moon illumination of Tycho area. Arrow shows crater Heinsius A. 
The small crater Heinsius A which exhibited the radar enhancement is the bright spot indicated by the arrow.

Also, it is known that during a lunar eclipse the rayed craters exhibit a different cooling rate than the rest of the lunar surface. The most recent measurements of a lunar eclipse in December 1964, show that many other craters also show this anamolous cooling behavior. One of the newly discovered "thermalanamolous" craters has been tentatively identified as Heinsius A, the crater which was bright under full moon and showed a radar enhancement. The optical, thermal, and radar results can be explained by localized areas of bare, exposed, and compact rock.

In conclusion, lunar radar echoes were examined in delay and frequency in a manner such that returns were reflected from localized areas on the lunar surface. The mapping of these returns showed that young and rayed craters have enhanced radar scattering. The craters which show these radar enhancements always appear bright on the full moon and from the latest infrared results, appear to be anamolous in that regime also. The results at all wavelengths can be explained if these are localized areas of bare, exposed, and compacted rocks.
Although this paper concerned the radar behavior of young and rayed craters it should be noted that the mountainous regions were shown to reflect more power than the maria regions. The enhancements were much less modest than those for the craters. For example, mountainous regions reflected $1 \frac{1 / 2}{2}$ to 2 times as much power as the maria region, while scattering enhancements of craters show they reflected up to 10 or 20 times as much power as other areas on the moon.

\section{Discussion Following Thompson's Paper}

T. Hagfors: What reflection law did you use?

T. W. Thompson: An empirical law based on observation from Millstone Hill at $440 \mathrm{Mc} / \mathrm{s}$, using a 100 $\mu$ sec pulse length.

C. Sagan: Have you looked to see whether there is any systematic difference between the scattering behavior of craters without central peaks?

T. W. Thompson: I have not looked specifically for this effect.

C. Sagan: Such a correlation might be expected.

(Paper 69D12-620)

\title{
Interpretation of the Angular Dependence of Backscattering From the Moon and Venus
}

\author{
Petr Beckmann
}

Electrical Engineering Department, University of Colorado, Boulder, Colo.

and

W. K. Klemperer

Central Radio Propagation Laboratory, National Bureau of Standards, Boulder, Colo.

\begin{abstract}
A previously derived formula, expressing the variation of the mean power backscattered from the rough surface of a planet with the delay time or angle of incidence by taking into account both the composite roughness of the surface and shadowing effects, is checked against new data from the Moon and Venus at five different wavelengths. The agreement with the lunar data is very good and leads to certain conclusions on the nature of the lunar surface. The data on Venus are also in good agreement and indicate that its surface is smoother than that of the Moon, but with an abundance of small structure.
\end{abstract}

\section{Introduction}

The problem of extracting information on the lunar and planetary surfaces from the measurements of radar backscatter from these surfaces has been the subject of many studies in recent years [cf. bibliography given by Evans, 1965a]. Until recently, no theory free of arbitrary and unnatural assumptions could provide a fit to the measured curves of backscattered power versus delay time (i.e., angle of incidence) and in the absence of this basic agreement the conclusions drawn from the measured curves must necessarily be doubtful.
In the present paper we hope to show that very good agreement with the measured curves may be obtained over the entire range of delay time by applying basic Kirchhoff theory and refining it to include two important effects: composite roughness and shadowing.

For radar backscatter from the Moon or a planet the pertinent geometry may be seen from figure 1 . A radio pulse of duration $T_{P}$ traveling with velocity $c$ illuminates an annular ring of area $A$ on the surface of the planet. This area is easily shown independent of position and equal to $A=2 \pi a c T_{p}$ with $a$ the radius of the planet. The angle of incidence $\theta$ changes from $0^{\circ}$ to $90^{\circ}$ as the pulse travels from the subterrestrial 